\title{
Antidepresivos y Suicidio: Una polémica actual
}

\author{
JUAN CARLOS MARTÍNEZ A. ${ }^{1}$, CLAUDIA GONZÁLEZ L. ${ }^{1}$ \\ 1. Psiquiatra de Niños y Adolscentes. Servicio de Psiquiatría, Hospital Naval Almirante Nef, Viña del Mar.
}

\begin{abstract}
Antidepressives and Suicide

Antidepressives have shown historically their utility in treatment of depression, confirmed not only in clinical studies, but also in the day-to-day medical practice. The treatment of depression is measured by reduction of suicide attempts and mortality associated to suicide, both conditions generally present in this disorder. During the last years, FDA has not allowed the use of antidepressives in children and adolescents due to increase in suicidal ideation, a fact observed in some studies with methodological incongruences and lack of significant differences with placebo. It is not possible to attribute direct responsibility to antidepressives because depression includes the diagnostic criteria of suicidal ideation reduction of suicide rate supports the effectiveness of these medications.

(Key words: antidepressives, suicide, infant psychiatry).

Rev Chil Pediatr 2007; 78 (5): 461-471
\end{abstract}

\section{RESUMEN}

Históricamente los antidepresivos han demostrado su utilidad en el tratamiento de la depresión, hecho confirmado no sólo en estudios clínicos, sino en la práctica clínica diaria. El tratamiento para la depresión se traduce en la reducción de los intentos y la muerte por suicidio, condición muchas veces inherentes a la depresión. Durante los últimos años, la FDA ha prohibido el uso de los antidepresivos en psiquiatría infanto-juvenil dado el incremento de la ideación suicida, hecho que se fundamenta en estudios metodológicamente insuficientes, en su mayoría no publicados, sin evidenciarse en ninguno de ellos diferencias con el placebo. No es posible atribuir responsabilidad directa a los antidepresivos puesto que de por sí la depresión cursa con ideación suicida, por el contrario, la reducción en las tasas de suicidio respalda la efectividad de este tipo de medicamentos.

(Palabras clave: antidepresivos, suicidio, psiquiatría infantil).

Rev Chil Pediatr 2007; 78 (5): 461-471

Trabajo recibido el 13 de junio de 2006, devuelto para corregir el 30 de enero de 2007, segunda versión el 5 de marzo de 2007, aceptado para publicación el 2 de junio de 2007. 


\section{Introducción}

La enfermedad mental en la infancia y adolescencia posee una relevancia especial y una trascendencia que supera a la mayoría de las enfermedades. Potencialmente un trastorno mental a temprana edad puede hipotecar la individualidad y la dimensión social de la persona llevando incluso al suicidio.

Según el informe mundial sobre la violencia y la salud de la Organización Mundial de la Salud (OMS, 2002), el suicidio (del latín: sui [de si mismo], y caedere [matar]), da cuenta de la primera causa de muerte por violencia entre la población general $(49,1 \%$ por suicidio, $31,3 \%$ por homicidios y $18,6 \%$ por acciones bélicas), falleciendo por esta razón una persona cada 38 a 40 segundos alrededor del mundo ${ }^{1}$. En niños, el suicidio corresponde a la quinta causa de muerte entre los 5 y 14 años, y a la tercera entre los 15 y 24 años $^{2}$. Si se considera un rango más estrecho (10 a 14 años), el suicidio ocupa el tercer lugar entre las causas de mortalidad ${ }^{3}$.

Si bien en la edad infanto-juvenil, el suicidio tiene una incidencia anual relativamente baja de 8 por 100000 al año, tanto la ideación como los intentos suicidas presentan una elevada prevalencia $^{3}$. Cada año, $19 \%$ de los adolescentes (15 a 19 años) tienen ideación suicida y 9\% intentan suicidio ${ }^{3}$.

Entre los jóvenes suicidas, los trastornos psiquiátricos presentan una alta prevalencia; de hecho, en el $90 \%$ de las autopsias psicológicas, es posible encontrar un diagnóstico psiquiátri$\mathrm{co}^{3,4}$. Ente ellos, la depresión es considerada la principal causa de suicidio, incrementando en más de 20 veces dicho riesgo en relación a la población general ${ }^{5}$.

La enfermedad depresiva en niños tiene una prevalencia que fluctúa entre un 2 a $5 \%$, alcanzando cifras más altas a medida que se acerca a la adolescencia ${ }^{3}$, explicando el $76 \%$ de las muertes por suicidio en este grupo etario ${ }^{6}$. Si bien un 2 a $8 \%$ de los niños depresivos cometería suicidio en una década de observación ${ }^{7-9}$, un importante número (60-70\%) de los pacientes depresivos experimentan ideación suicida ${ }^{10}, \mathrm{y}$ 35 a $50 \%$ intentará suicidio ${ }^{8,11}$.

Durante el último cuarto de siglo, el suicidio entre los jóvenes ha emergido como un importante problema de salud pública. En muchos países, el suicidio -en este grupo de pacientesse ha incrementado notablemente, llegando a triplicarse desde los años 60' a la década de los 90', particularmente en el sexo masculino ${ }^{12,13}$.

Para Bridge y cols (2006), se ha avanzado bastante en el conocimiento de la fenomenología y de los factores de riesgo de las conductas suicidas y suicidio del adolescente ${ }^{14}$. Entre estos destacan: 1) enfermedades mentales o físicas; 2) alteraciones de la personalidad; 3) factores familiares; 4) factores biológicos; 5) aprendizaje externo o "contagio"; 6) acceso a agentes letales y 7) pobre intervención de la enfermedad o mal manejo clínico.

Por ser el suicidio un acto en cuya realización influye la conjunción de múltiples factores, es muy difícil atribuir exclusiva responsabilidad a un factor específico. Como se indica en la tabla 1, cada condición biológica, psicológica o social, agrega un riesgo adicional para suicidio;

Tabla 1. Factores de riesgo para suicidio en adolescentes víctimas de suicidio

\begin{tabular}{lrcc}
\hline & Mujeres & $\begin{array}{c}\text { Riesgo relativo } \\
\text { Hombres }\end{array}$ & Total \\
\hline Enfermedad psiquiátrica & $4,3-11,2$ & $5-15,6$ & $4,8-22,0$ \\
Enfermedad del ánimo & $14,2-25,2$ & $9,1-11,3$ & $8,2-13,0$ \\
Abuso de sustancias & $3,1-12,2$ & $7,2-11,9$ & $4,1-12,7$ \\
Trastornos ansiosos & $0,7-2,8$ & $3,2-13,0$ & $2,3-4,2$ \\
Trastornos conductuales & $1,4-3,3$ & $3,5-7,4$ & $3,5-6,1$ \\
Intento previo suicidio & $37,2-68,3$ & $34,3-52,8$ & $50,6-89,3$ \\
\hline
\end{tabular}

* Se indican los hallazgos de varios estudios. El rango inferior para cada parámetro indica el riesgo relativo más bajo reportado en los estudios revisados para dicha condición psiquiátrica. El rango superior indica el riesgo relativo más alto reportado en los estudios revisados para dicha condición psiquiátrica ${ }^{7,14-16}$. 
por ejemplo, el riesgo relativo de suicidio se incrementa hasta 22 veces cuando se padece una enfermedad psiquiátrica, y el antecedente previo de suicidio puede incrementar el riesgo en un amplio rango que va entre las 9 a 60 veces, llegando incluso a casi 90 veces según algunos reportes ${ }^{7,14-17}$.

Tras un intento de suicidio, el riesgo de repetición más alto se produce entre los primeros 3 a 6 meses posteriores, manteniéndose particularmente elevado durante los 2 años siguientes ${ }^{18,19}$.

Por otra parte, estudios de autopsias psicológicas demuestran que sobre el $70 \%$ de los jóvenes suicidas presentaban múltiples comorbilidades psiquiátricas, lo que incrementa notablemente el riesgo autolítico existiendo una relación directamente proporcional entre número de patologías asociadas y probabilidad de cometer suicidio. Particular gravedad confiere la comorbilidad "trastorno del ánimo, trastorno de la conducta y abuso de drogas", siendo esta la más "potente" combinación para el caso de los adolescentes ${ }^{14,19}$.

Las características de la personalidad, como la impulsividad, agresividad, irritabilidad, poca tolerancia a la frustración, patrones rígidos cognitivos, escasa capacidad adaptativa, pobre resiliencia (facultad de hacer frente a la adversidad y construir positivamente sobre ella), también impactan negativamente en el riesgo suicida. Los trastornos de la personalidad, se presentan en aproximadamente uno de cada tres adolescentes muertos por suicidio, incrementando en 3 veces el riesgo de suicidio (para el caso de todos los tipos de personalidades), y en 8,5 veces para las denominadas personalidades "cluster B" (antisocial, borderline, histriónico, narcisista $)^{14,20}$.

Otras condiciones asociadas son los de tipo familiar y medio ambiental. Entre ellos cobra valor la psicopatología parental (depresión, abuso de sustancias, conductas antisociales), la historia familiar de suicidio, la inestabilidad o conflictiva intrafamiliar, la muerte de uno de los padres o el divorcio, y la presencia de cualquier tipo de violencia. Intervienen además, la relación interpersonal con pares, el apoyo del sistema escolar, la disponibilidad y acceso a algún agente letal (armas, fármacos), entre otros.
Un factor de riesgo suicida -no ajeno de controversias- es el uso de los antidepresivos (ATD), lo que precisamente ha motivado la presente revisión.

El tema suicidio - ATD, ha estado presente desde los inicios de la farmacopea ATD. Por una parte, la toxicidad cardiovascular de una sobre dosis con ATD tricíclicos (clomipramina, amitriptilina, imipramina), y por otra el conocimiento de que el período inicial de la terapia antidepresiva es un período crítico para cometer suicidio, hacían del tratamiento una situación de cuidado.

El primer período de tratamiento, alertaba al clínico toda vez que los primeros signos de mejoría correspondían a la disminución del retardo psicomotor y al aumento en los niveles de energía; y entre los últimos, los sentimientos de baja autoestima, la desesperanza, la culpa y la suicidalidad $^{21,22}$. Por ello, existía una ventana en que los pacientes sentirían "mayor fuerza" sin disminuir la intencionalidad autoagresiva o el deseo de muerte.

Hasta la actualidad, y considerando que todo paciente con depresión tiene riesgo de suicidio, deben adoptarse medidas preventivas, especialmente durante las primeras semanas de tratamiento, período en el cual mejora el impulso mientras persiste el ánimo depresivo y la ideación suicida. Cuando el paciente aparentemente empieza a estar mejor, el riesgo de suicidio puede volverse mayor y el cuidado debe ser más estrecho.

Desde comienzos de los años 80' a la fecha, se han introducido una nueva clase de ATD: los inhibidores selectivos de la recaptación de serotonina (ISRS) como la fluoxetina, sertralina, citalopram, escitalopram, paroxetina, fluvoxamina, entre otros, cuya llegada significó un importante cambio en el manejo de la depresión. Pese a no ser más efectivos que los tricíclicos y a demorar un par de semanas en lograr sus efectos terapéuticos, su perfil resulta más favorable desde el punto de vista cardiovascular, siendo muy difícil un suicidio por sobredosis ${ }^{21,22}$.

Pese a la "benignidad" asignada inicialmente, los ISRS no escaparon a la polémica del suicidio. En 1990, Teicher y cols, reportaron los casos de 6 pacientes libres de ideación suicida tratados con fluoxetina, que desde el tercer día 
hasta 3 meses después de suspendida la medicación presentan ideación suicida, destacando que ninguno de ellos había presentado previamente síntomas de ese tipo en respuesta al tratamiento con otras drogas psicotrópicas ${ }^{23}$.

Trece años más tarde, en diciembre del 2003 la British Medicines and Healthcare Products Regulatory Agency (MHRS) sugiere el cese del uso de ATD en menores de 18 años, a excepción de la fluoxetina, basado en 3 estudios (dos de los cuales no están publicados), que relaciona a los ATD con incremento de conductas suicidas en niños y adolescentes $^{24}$. El único estudio publicado, comparaba la incidencia "de novo" de ideación suicida en pacientes con paroxetina versus placebo, encontrándose un promedio de 3,7\% (14/378 pacientes) y $2,5 \%(7 / 285)$ respectivamente, sin diferencias estadísticamente significativas entre ambos grupos $(p=0,5)^{25}$. En estos estudios, la definición de suicidalidad resulta ambigua, incluyendo conductas como ideación suicida, autoagresiones e intentos de autoeliminación.

Por otra parte, en los Estados Unidos, la Food and Drugs Administration (FDA), en una extensa revisión que incluyó 24 estudios con un total de 4100 pacientes (14 estudios en depresión, el resto en cuadros ansiosos y en déficit atencional hiperactivo), señala que los individuos medicados con ATD, tuvieron 1,8 veces más eventos suicidas (ideación, intento, autoagresión) en comparación con el placebo (3,8 vs 2,1\%); tampoco hubo reporte de suici$\operatorname{dios}^{25,26}$. En la tabla 2, se observa la prevalencia de suicidalidad para ATD y placebo recogido de algunos de los estudios señalados.
En marzo del 2004, la FDA sugirió restringir y vigilar el uso de ATD a los pacientes adultos, ante la posibilidad de empeorar los síntomas depresivos y la ideación suicida, extendiendo la "duda" a la fluoxetina, sertralina, paroxetina, fluvoxamina, citalopram, escitalopram, bupropion, venlafaxina, nefazodona y mirtazapina. Finalmente en octubre del 2004, tras evaluar 26 estudios, la FDA aplicó el black box a todos y a los futuros antidepresivos en menores de 18 años de edad. Sin embargo, nuevamente en ninguno de los 26 estudios se evidenció algún caso de suicidio. Por otra parte, la gran mayoría de los trabajos no han sido "mostrados" a la comunidad científica, desconociéndose la rigurosidad metodológica y estadística ${ }^{25,26}$.

En febrero del 2005, el black box original fue cambiado y acotado por la FDA, precisando que el uso de ISRS produciría incremento de la ideación y de conductas, no así de suicidio propiamente tal. Posteriormente, en junio de ese mismo año, el mismo organismo americano advierte nuevamente la posibilidad de incrementar la suicidalidad en adultos ${ }^{25,26}$.

Los profesionales de la salud mental hemos sido testigos presenciales del cómo el "alerta" de la FDA ha limitado el quehacer terapéutico, generando confusión y temor respecto al modo de enfrentar un paciente con el diagnóstico de depresión. De hecho, según algunos reportes, desde la advertencia de la FDA, los médicos en general han tendido a disminuir la prescripción de dichos fármacos ${ }^{27}$. Por lo demás, no es infrecuente ver que los padres de nuestros pacientes - cada vez más informados- critiquen abiertamente nuestra decisión terapéutica, de-

Tabla 2. Suicidalidad según antidepresivo vs placebo

\begin{tabular}{lccccc}
\hline & \multicolumn{5}{c}{ Suicidalidad (\%) } \\
\cline { 2 - 6 } Antidepresivo & $\mathbf{n}$ & Antidepresivo & Placebo & Diferencia & p \\
\hline Fluoxetina & 458 & 3,6 & 3,8 & $-0,2$ & $0 ; 90$ \\
Setralina & 373 & 2,7 & 1,1 & $+1,6$ & 0,45 \\
Citalopram & 418 & 8,9 & 7,3 & $+1,6$ & 0,40 \\
Paroxetina & 663 & 3,7 & 2,5 & $+1,2$ & 0,50 \\
Venlafaxina & 334 & 2,0 & 0,0 & $+2,0$ & 0,25 \\
\hline
\end{tabular}

Modificado de Brent y cols (2004)26

*Suicidalidad: ideación, autoagresiones, intentos suicidas. (+): incremento del riesgo suicida; (-) disminución del riesgo suicida. 
cidiendo unilateralmente no dar o suspender la medicación, para de ese modo "proteger a sus hijos" del uso de los ATD, que para quienes trabajamos en trastornos del ánimo resulta un contrasentido.

El objetivo de la presente revisión es mostrar la evidencia a favor y en contra de los ATD en relación a una probable suicidalidad, para ello hemos realizado una revisión en MEDLINE de los estudios relacionados con el tema. Para la selección de los artículos, hemos utilizado las palabras claves "antidepressant", "suicide", "depression" y las combinaciones "antidepressant and suicide", "antidepressant and child", "antidepressant and adolescence".

\section{Desarrollo}

Los estudios que relacionan ATD/suicidalidad demuestran que de cada 100 pacientes, 2 o 3 tienen un riesgo incrementado por sobre placebo en cuanto pensamiento suicida, intento suicida o autoagresiones. Al ser la mayoría de los estudios "desconocidos" desde el punto de vista metodológico, la multi-factorialidad del suicidio, ¿permite asignar un rol suicido-génico a los ATD, sin tener la certeza de haber depurado los factores biológicos, psicológicos y sociales que impactan en la concreción de dicho acto?; los grupos ¿serán comparables?, ya que desde el punto de vista ético queda la duda si los investigadores habrán asignado placebo a un paciente gravemente depresivo, con intentos de suicidio o ideación suicida actual.

Los estudios señalados por la FDA y por la MHRS no considerarían importantes factores como: la condición clínica previa y actual del paciente (falta absoluta de respuesta, respuesta parcial, remisión, recaída, etc), fracaso en la adherencia, discontinuación del tratamiento, uso de alcohol y drogas, grado de religiosidad, rasgos de personalidad, comorbilidad médica o psiquiátrica, antecedentes previos de suicidio, antecedentes psiquiátricos familiares, situación intrafamiliar e interpersonal, parámetros farmacocinéticos (como una excesiva metabolización que se traduce en un nivel sérico ATD subóptimo), tiempo transcurrido entre el inicio del tratamiento y la aparición de la ideación o del acto autolesivo, etc ${ }^{28}$; lo que implica una seria limitación metodológica.

Habitualmente los estudios con fármacos son realizados con un grupo "ideal", esto es, pacientes sin ideación suicida actual, sin comorbilidad médica o psiquiátrica, sin trastornos de la personalidad, con una buena red de apoyo, etc, lo que dista de la realidad y del común de los consultantes, por lo que habría que preguntarse: ¿qué ocurre con el paciente "real" y el uso de los ATD? En psiquiatría infanto-juvenil, es precisamente el intento de suicidio o la autogresión lo que da el alerta a los padres de que sus hijos necesitan la ayuda de un profesional. Al respecto, precisamente indicamos a estos pacientes ISRS dada la buena tolerancia y baja toxicidad ante el riesgo de una sobredosis voluntaria.

Como el predictor más "potente" de un futuro intento suicida es el antecedente de suicidalidad previa, se podría atribuir injustamente dicho rol al ATD. Sobre 2/3 de quienes han intentado autoeliminación repetirán dicha conducta en el tiempo. Más aún, cada intento suicida aumenta el riesgo de recurrir con métodos más agresivos y eficaces ${ }^{28}$.

Uno de los ATD más cuestionados ha sido venlafaxina, un antidepresivo dual, con acción sobre receptores serotoninérgicos y noradrenérgicos. Este fármaco goza de gran prestigio en el tratamiento de los casos de depresión severa, siendo habitualmente utilizado por los médicos en aquellos casos más complejos, que de por si tienen mayor riesgo suicida. Un estudio realizado por Mines y cols el año 2005, confirma que el "más criticado" de los ATD es el fármaco reservado para los pacientes más complejos. Los pacientes tratados con venlafaxina versus los grupos tratados con fluoxetina y citalopram tenían respectivamente 2,75 y 2,43 veces más antecedentes de conductas suicidas; 6,2 y 4,3 veces más antecedentes de hospitalización por depresión; y 6,55 y 3,1 veces mayor probabilidad de haber recibido 2 o más ATD durante el último año ${ }^{29}$. Por lo tanto, eran pacientes con un alto riesgo de recurrencia suicida.

Con los antecedentes expuestos, y a nuestro modo de ver, la recomendación de no emplear ATD por un eventual incremento de la suicida- 
lidad equivale a desaconsejar el uso de antibióticos, broncodilatadores o antihistamínicos en el tratamiento de la neumonía, asma bronquial o urticaria, respectivamente, argumentando que dichos fármacos pueden producir tos, disnea o prurito, o exacerbar la patología de base. En realidad se trata de síntomas inherentes a la enfermedad, cuya exacerbación puede deberse a la severidad de la enfermedad o a que los fármacos utilizados, o su dosificación, no son suficientes para controlar los síntomas en cuestión. En ningún caso la conclusión sería abstenerse de usar esos medicamentos ${ }^{30}$.

Según reportes, quienes intentan suicidio reciben en un porcentaje mayor terapia ATD comparado con quienes no han intentado suicidio, aunque esto sería atribuible a que el uso de ATD es un "marcador" de severidad depresi$\mathrm{va}^{31}$. El riesgo de intento suicidio grave durante un seguimiento de 6 meses de tratamiento ATD es menos de 1 por 1000 adultos, y menos de 1 por 300 adolescentes $^{32}$.

En el caso hipotético de que realmente los ISRS incrementasen la ideación suicida, esto tampoco sería una causal prohibitiva para no tratar la depresión mayor puesto que 1 de 6000 niñas y 1 de 400 niños con ideación presente cometen suicidio $^{33}$. Así mismo, la relación entre intentos suicidas entre los jóvenes y el suicidio es de 100 a 200:134.

En la gran mayoría de los estudios en que se basa la FDA y que han sido mostrados a la comunidad científica, el seguimiento se limita a menos de 12 semanas, por lo que, a largo plazo es difícil evidenciar el impacto del tratamiento. Al respecto, y tal como lo plantea Jick y cols ${ }^{35}$, a medida que se prolonga un tratamiento ATD, el riesgo de intento y el suicidio se van reduciendo considerablemente. Comparado con los pacientes que llevaban más de 90 días tomando ATD, entre el primer y noveno día de tratamiento la probabilidad de desarrollar un acto suicida no fatal era 4 veces superior; entre los 10 y 29 días, 3 veces superior; y, entre los 30 y 89 días de tratamiento el riesgo se elevaba a 1,5 veces. Del mismo modo, comparado con quienes llevaban más de 90 días de tratamiento, el riesgo de muerte por suicidio era 38 veces más alto entre los días primeros 9 días de tratamiento, reduciéndose drásticamente a 5,1 veces en- tre los días 10 y 29 , y a 2 veces entre los 30 y 89 días de tratamiento ${ }^{35}$.

A pesar del notable incremento del suicidio adolescente hasta la década de los 90', existe una fuerte evidencia de que los ATD resultan útiles en la reducción del riesgo autolítico. Tal como se observa en la tabla 3, hay países en que la tasa de suicidio se ha incrementado y otros en que ha tendido al estancamiento o a la reducción ${ }^{13,14}$. Para estimar con certeza el impacto de los ATD, habría que ver la reducción de los comportamientos suicidas en los pacientes depresivos tratados y no tanto lo observado en la población general.

Al respecto, desde los inicios de la terapéutica en psiquiatría se ha observado una progresiva y significativa disminución en las tasas de suicidio entre los pacientes depresivos. Así, en la era "pre-terapéutica" (1900-1939), en la era de la "terapia electroconvulsiva" (1940-1959), y en la "era ATD" (1960-1992), las tasas se redujeron de modo progresivo $(6,3,5,7$ y 3,3 por cada 1000 pacientes) ${ }^{36}$. Del mismo modo, desde 1985 a 1999 la tasa de suicidio en los Estados Unidos disminuyó de 12,4 x 100000 habitantes a 10,7 x $100000(-13,5 \%)$, mientras que la prescripción de los ATD, fundamentalmente ISRS se incrementó 4 veces. La declinación fue más pronunciada en mujeres que en hombres, coincidente (pero no atribuible exclusivamente) con el hecho que las mujeres recibieron 2 veces más ATD que los hombres ${ }^{37}$. Por su parte, en adolescentes, el doble en el uso de ATD se asoció a una disminución de 0,23 suicidios cada 100000 adolescentes por año ${ }^{38}$.

Las consultas por episodios depresivos tanto mono o bipolares se han incrementado notablemente durante la última década y con ello la prescripción ATD, por lo que, la exposición a dichos fármacos resulta evidentemente mayor. Si consideramos el riesgo suicida del depresivo, un análisis ligero de dichos casos podría atribuir injustamente responsabilidad a los $\mathrm{ATD}^{39}$.

Por su parte, estudios señalan que un número importante de suicidas consultó médico el mes previo a su muerte, pese a lo cual, la mayoría no recibió un esquema terapéutico ${ }^{40-42}$, estando una muy pequeña cantidad de pacientes en tratamiento al momento de su muerte ${ }^{15}$. De acuerdo con lo señalado por Gray y cols, 
sólo un 1\% de los suicidas jóvenes se encontraban bajo tratamiento en el sistema público de salud, y en un 3\% fue posible detectar psicotrópicos en plasma durante la autopsia ${ }^{43}$. Otros autores, concuerdan con que el $2 \%$ de los jóvenes suicidas recibe tratamiento ATD $^{44-46}$.
Prescribir un ATD no asegura que se continúen las indicaciones de tratamiento; la no adherencia de los ISRS en la población general fluctúa entre el 15 a $20 \%{ }^{47}$, y la falta de respuesta para estos fármacos es alrededor del 20 a $30 \%{ }^{48}$. Considerando estas cifras, resulta

Tabla 3. Tasa suicidio país según década y grupo etario ${ }^{14}$

\begin{tabular}{|c|c|c|c|c|c|c|}
\hline \multirow[b]{2}{*}{ País } & \multicolumn{3}{|c|}{ edad 5 a 14 años } & \multicolumn{3}{|c|}{ edad 15 a 24 años } \\
\hline & 1990 & 2000 & Variación & 1990 & 2000 & Variación \\
\hline \multicolumn{7}{|l|}{ Europa del Este } \\
\hline Estonia & 1,3 & 0,5 & $-0,8$ & 17,2 & 18,1 & $+0,9$ \\
\hline Rusia & 1,4 & 2,7 & $+1,3$ & 17,8 & 33,7 & $+15,9$ \\
\hline Latvia & 1,9 & 1,9 & 0 & 13,6 & 17,9 & $+4,3$ \\
\hline Hungría & 1,7 & 0,9 & $-0,8$ & 14,3 & 10,7 & $-3,6$ \\
\hline Polonia & 0,6 & 0,9 & $+0,3$ & 9,6 & 11,4 & $+1,8$ \\
\hline República Checa & 0,4 & 1,0 & $+0,6$ & 8,7 & 10,5 & $+1,8$ \\
\hline \multicolumn{7}{|c|}{ Europa del Norte } \\
\hline Finlandia & 0,6 & 0,3 & $-0,3$ & 31,3 & 19,9 & $-11,4$ \\
\hline Noruega & 0,8 & 0,7 & $-0,1$ & 14,4 & 15,5 & $+1,1$ \\
\hline Suecia & 0,5 & 0,2 & $-0,3$ & 10,1 & 8,7 & $-1,4$ \\
\hline Alemania & 0,4 & 0,4 & 0 & 9,5 & 7,7 & $-1,8$ \\
\hline Holanda & 0,4 & 0,3 & $-0,1$ & 5,9 & 5,7 & $-0,2$ \\
\hline \multicolumn{7}{|l|}{ Habla Inglesa } \\
\hline Nueva Zelandia & 0,4 & 0,7 & $+0,3$ & 23,1 & 18,2 & $-4,9$ \\
\hline Australia & 0,2 & 0,3 & $+0,1$ & 15,9 & 13,3 & $-2,6$ \\
\hline Canadá & 0,8 & 1,1 & $+0,3$ & 15,0 & 13,0 & $-2,0$ \\
\hline Estados Unidos & 0,8 & 0,8 & 0 & 13,2 & 10,2 & $-3,0$ \\
\hline Irlanda & 0,6 & 0,2 & $-0,4$ & 9,3 & 16,1 & $+\quad 6,8$ \\
\hline Reino Unido & 0,03 & 0,1 & $+0,07$ & 7,2 & 6,8 & $-0,4$ \\
\hline \multicolumn{7}{|l|}{ Europa Católica } \\
\hline Austria & 0,3 & 0,9 & $+0,6$ & 15,4 & 13,9 & $-\quad 1,5$ \\
\hline Francia & 0,3 & 0,6 & $+0,3$ & 9,3 & 7,9 & $-\quad 1,4$ \\
\hline España & 0,4 & 0,2 & $-0,2$ & 4,5 & 4,7 & $+0,2$ \\
\hline Portugal & 0,3 & 0,0 & $-0,3$ & 5,4 & 2,4 & - 3,0 \\
\hline Italia & 0.3 & 0,2 & $-0,1$ & 4,0 & 4,2 & $+\quad 0,2$ \\
\hline \multicolumn{7}{|l|}{ Asia } \\
\hline Singapur & 0,7 & 1,2 & $+0,5$ & 10,4 & 7,1 & $-3,3$ \\
\hline Japón & 0,3 & 0,6 & $+0,3$ & 7,0 & 11,5 & $+4,5$ \\
\hline Hong Kong & 0,2 & 1,4 & $+1,2$ & 7,1 & 6,5 & $-0,6$ \\
\hline \multicolumn{7}{|l|}{ Latinoamérica } \\
\hline Argentina & 0,6 & 0,6 & 0 & 5,1 & 8,7 & $+\quad 3,6$ \\
\hline México & 0,2 & 0,5 & $+0,3$ & 3,1 & 5,4 & $+2,3$ \\
\hline \multicolumn{7}{|l|}{ Otros } \\
\hline Israel & 0,2 & 0,5 & $+0,3$ & 5,0 & 6,5 & $+\quad 1,5$ \\
\hline
\end{tabular}

Modificado de Bridge y cols (2006) ${ }^{14}$. *Tasas por 100 000/año. 
fundamental la pesquisa de los casos en que fracasa un ATD o falla la adherencia, debiendo fortalecerse el vínculo terapéutico, particularmente en quienes tengan un elevado riesgo de suicidalidad. Precisamente, un factor importante que agrava el problema es la baja adherencia al tratamiento revelado en estudios toxicológicos. En pacientes muertos por suicidio un muy bajo porcentaje de quienes recibían ATD tenían niveles plasmáticos pesquizables, lo que permite concluir que el suicidio ocurría en quienes no respetaban las indicaciones o en quienes habían suspendido el tratamiento. Gray y cols, en 49 adolescentes suicidas de Utah, encuentran que el $24 \%$ estaba en tratamiento antidepresivo, sin embargo, en ninguno de ellos fue encontrado registro plasmático post-mortem para antidepresivos ${ }^{49}$. Otros autores encuentran cifras entre un 8 a 20\% para reporte positivo de ATD en sangre al momento de su muerte ${ }^{50-53}$.

\section{Posibles motivos que influirían en el incremento de la suicidalidad}

\section{Viraje Farmacológico y Fases Mixtas}

El trastorno bipolar (TB) es habitualmente mal diagnosticado como depresión unipolar ${ }^{54}$, recurriéndose regularmente a los ATD como parte importante de la terapéutica, los cuales están contraindicados en bipolares como monoterapia, debiendo siempre asociarse a un estabilizador del ánimo como el ácido valproico, carbamazepina, carbonato de litio, oxcarbazepina, etc. La razón de lo anterior, es el riesgo de viraje desde la fase depresiva a la maníaca, con descompensaciones bruscas del cuadro clínico.

Un factor que podría influir en un eventual incremento de la suicidalidad, es precisamente el paso de la depresión a la manía, principalmente a manía disfórica o irritable. De hecho, una muy baja tasa de pacientes con TB recibe tratamiento con estabilizadores al momento de su muerte ${ }^{55}$. Al respecto, Wilens y cols (2003), señalan que un $7 \%$ de los pacientes pediátricos tratados por depresión mayor y trastorno obsesivo-compulsivo desarrollaron manía, y $10 \%$ un cuadro psicótico ${ }^{56}$. Otros autores reportan tasas de agresividad, conductas autoinjuriantes e ideación homicida en pacientes que desarrolla- ron manía con una frecuencia del 77\%, 20\% y $6 \%$ respectivamente ${ }^{57}$.

Por otra parte, los bipolares no diagnosticados tendrían 4 veces más riesgo de intentar suicidio, y 50\% más probabilidad ser hospitalizado al compararlos con quienes no tienen una enfermedad bipolar ${ }^{58}$.

\section{Akatisia}

La akatisia corresponde a "una sensación de inquietud, impaciencia, intranquilidad, ansiedad, insomnio o disconfort generalizado", y está fuertemente asociada a abandono precoz de tratamiento. La akatisia secundaria a los ATD tendría una incidencia que varía entre un 9,8 a $25 \%{ }^{59}$, y a menudo se presenta durante los primeros 10 a 14 días de tratamiento, siendo comúnmente ignorada o confundida con ansiedad o con empeoramiento de la condición origi$\mathrm{nal}^{60}$. Pese a la evidencia, no es clara la relación o la influencia de la akatisia en el suicidio, aunque una probable relación estaría basada en la sensación desagradable, inquietud, insomnio secundario, y en la baja adherencia al tratamiento ATD que se desprende de su sintomatología.

\section{Discontinuación del antidepresivo}

La discontinuación abrupta de los ATD pueden producir un conjunto de síntomas inmediatamente después de la suspensión medicamentosa. Para hablar de "síndrome de discontinuación”, los síntomas deben haberse presentado previo a la suspensión y no deben ser atribuibles a otras causas. Síntomas comúnmente asociados son mareos, ansiedad, parestesias, irritabilidad, síntomas gripales (malestar general, rinorrea, mialgias, náuseas, vómitos, diarrea); síntomas menos comunes son alucinaciones hipnagógicas, hipomania, agitación psicomotora, con reportes aislados de ideación suicida. Como parte del concepto de este síndrome está el hecho que la reinserción del tratamiento mejora rápidamente los síntomas señalados ${ }^{61}$.

\section{Postura de la American Academy of Child and Adolescent Psychiatry}

La American Academy of Child and Adolescent Psychiatry (AACAP) ha sugerido 
a la FDA no aplicar el black box warning a todos los ATD en depresión infantil basado en los siguientes postulados ${ }^{62}$ :

a) Existe una fuerte evidencia de la eficacia de los ATD en depresión mayor.

b) La exacerbación de eventos suicidas no tienen fuerza estadística, y si así fuera, podrían ser monitorizados.

c) Según FDA sólo 78/4 400 (2-3\%) niños y adolescentes, incrementaron ideación suicida o conductas autolesivas, sin ningún reporte de suicidio.

d) El costo de la depresión para la vida del paciente es mayor que un incremento del $2 \%$ de la suicidalidad.

e) Ante la posibilidad de ideación o conductas suicidas frente al inicio de un tratamiento ATD o secundario al cambio de las dosis se recomienda: control telefónico/personal frecuente (primer mes) como entregar una lista de síntomas de alarma y buscarlos en los controles; estar alerta ante aparición o aumento de síntomas como ansiedad, crisis de pánico, agitación, akatisia, insomnio, irritabilidad, hostilidad (agresividad), impulsividad, hipomanía, y manía. Pese a lo anterior, no hay claridad causal entre estos síntomas y la ideación/conductas suicidas.

f) Si los síntomas son severos, de inicio brusco, o no estaban presentes en el cuadro inicial, considerar cambio de esquema terapéutico.

\section{Conclusiones}

Mientras más grave una depresión, mayor probabilidad consultar al médico y de ser tratada con uno o más ATD, eligiéndose -por lo general- los más modernos, cada vez más seguros, efectivos y potentes, lo que evidentemente establece una relación entre quienes usan ATD y el mayor riesgo suicida, que en muchos casos es atribuible a la severidad del cuadro clínico.

Los estudios que llevan a black box por parte de los organismos regulatorios norteamericanos y británicos, no gozan de una metodología rigurosa, y sus resultados no alcanzan significancia estadística. Los resultados sistemáticamente demuestran que cada 100 pacientes, 2 a
3 incrementan el riesgo de suicidalidad, cifras que no se comparan con la ideación y riesgo de suicida que acompaña a la enfermedad depresiva. En relación a esto, la FDA plantea que el riesgo de tratar una depresión es incrementar la suicidalidad en un $2 \%$ por sobre el placebo; cabría preguntarse entonces, ¿qué ocurriría si no se tratan a los pacientes?.

La observación en niños y adolescentes coincide en que prácticamente la totalidad de los suicidas no tomaba ATD pese a estar deprimidos, lo que sugiere una baja relación fármaco/ suicidio.

Por lo expuesto, creemos que la FDA ha tenido una actitud sobrevalorada en contra los ATD, sin considerar el efecto negativo para los médicos y particularmente para nuestros enfermos.

\section{Referencias}

1.- Informe mundial sobre la violencia y la salud. Washington, D.C., Organización Panamericana de la Salud, Oficina Regional para las Américas de la Organización Mundial de la Salud, 2003.

2.- Kochanek KD, Smith BL: Deaths: Preliminary data for 2002. National Vital Statistics Reports (CDC) 2004; 52: 1-48.

3.- Gibbons RD, Hur K, Bhaumik DK, Mann JJ: The relationship between antidepressant prescription rates and rate of early adolescent suicide. Am J Psychiatry 2006; 163: 1898-904

4.- Brent DA, Perder JA, Goldstein CE, et al: Risk factors for adolescent suicide: a comparison of adolecent suicide victims with suicidal inpatients. Arch Gen Psychiatry 1988; 45: 581-8.

5.- Harris EC, Barraclough B: Suicide as an outcome for mental disorders. A meta-analysis. Br J Psychiatry 1997; 170: 205-28.

6.- Moller HJ: Suicide, suicidality and suicide prevention in affective disorders. Acta Psychiatr Scand 2003; 418: 73-80.

7.- Shafii M, Lenarsky J, Derrick AM, Beckner C, Whittinghill JR: Comorbidity of mental disorders in the post-mortem diagnosis of completed suicide in children and adolescents. J Affect Disord 1988; 15: 227-33.

8.- Fombonne E, Wostear $G$, Cooper $V$, Harrington $R$, Rutter M: The Maudsley long-term follow-up of child and adolescent depresión, II: suicidality, criminality, and social dysfunction in adulthood. Br J Psychiatry 2001; 179: 218-23.

9.- Rao U, Weissman MM, Martin JA, Hammond RW: Childhood depression and risk of suicide: a preliminary report of a longitudinal study. J Am Acad Child Adolesc Psychiatry $1993 ; 32: 21-7$. 
10.- Shaffer D, Fisher P, Dulcan MK: The NIMH diagnostic interview schedule for children versión 2.3 (DISC-2.3): description, acceptability, prevalence rates, and performance in the MECA study. Methods for the epidemiology of child and adolescent mental disorders study. J Am Acad Child Adolesc Psychiatry 1996; 35 : 865-77.

11.- Kovacs M, Goldston D, Gatsonis C: Suicidal behaviors and childhood-onset depressive disorders: a longitudinal investigation. J Am Acad Child Adolesc Psychiatry 1993; 32: 8-20.

12.- Gould MS, Greenberg T, Velting DM, Shaffer D: Youth suicide risk and preventive interventions: A review of the past 10 years. J Am Acad Child Adolesc Psychiatry 2003; 42: 386-405.

13.- World Health Organization. Suicide rates and absolute numbers of suicide by country (2002). Available online: http://www.who.int/mental_health/prevention/suicide/ (accessed April 7, 2005).

14.- Bridge JA, Goldstein TR, Brent DA: Adolescent suicide and suicidal behavior. J Child Psychol Psychiat 2006; 47: 372-94.

15.- Shaffer D, Gould MS, Fisher P, et al: Psychiatric diagnosis in child and adolescent suicide. Arch Gen Psychiatry 1996; 53: 339-48.

16.- Brent DA, Baugher M, Bridge J, Chen T, Chiappetta L: Age- and sex-related risk factors for adolescent suicide. J Am Acad Child Adolesc Psychiatry 1999; 38: 1497-505.

17.- Martínez C, Rietbrock S, Wise L, et al: Antidepressant treatment and the risk of fatal and non-fatal self harm in first episode depression: nested case-control study. BMJ 2005; 330: 89.

18.- Goldston DB, Daniel SS, Reboussin DM, Reboussin BA, Frazier PH, Kelley AE: Suicide attempts among formerly hospitalized adolescents: A prospective naturalistic study of risk during the first 5 years after discharge. J Am Acad Child Adolesc Psychiatry 1999; 38: $660-71$

19.- Lewinsohn PM, Rohde P, Seeley JR: Adolescent suicidal ideation and attempts: Prevalence, risk factors, and clinical implications. Clin Psychol Sci Pract 1996; 3: 25-36.

20.- Brent DA, Johnson BA, Perper J, Connolly J, Bridge J, Bartle $S$ : Personality disorder, personality traits, impulsive violence, and completed suicide in adolescents. J Am Acad Child Adolesc Psychiatry 1994; 33: 1080-6.

21.- Lapid MI, Rummans TA: Evaluation and management of geriatric depression in primary care. Mayo Clin Proc 2003; 78: 1423-9.

22.- Remick RA: Diagnosis and management of depression in primary care: a clinical update and review. CMAJ 2002; 167: 1253-60.

23.- Teicher MH, Glod C, Cole JO: Emergence of intense suicidal preoccupation during fluoxetine treatment. Am J Psychiatry 1990; 147: 207-10.

24.- Möller HJ: SSRIs: Are the Accusations Justified?. World J Biol Psychiatry 2004; 5: 174-5.

25.- Goode E: British warning on antidepressant use for youth. The New York Times. December 11, 2003. p. A1.

26.- Brent DA, Birmaher B: British warnings on SSRIs questioned. J Am Acad Child Adolesc Psychiatry 2004; 43: 4.

27.- Rappaport N, Bostic JQ, Prince JB, Jellinek M: Treating pediatric depresión in primary care: doping with the patients's blue and mood and the FDA's black box. J Pediatr 2006; 148: 567-8.

28.- Rihmer Z, Akiskal H: Do antidepressants t(h)reat(en) depressives? Toward a clinically udicious formulation of the antidepressant-suicidality FDA advisory in light of declining national suicide statistics from many countries. J Affect Disord 2006; 94: 3-13.

29.- Mines $D$, Hill D, Yu H, Novelli L: Prevalence of risk factors for suicide in patients prescribed venlafaxine, fluoxetine, and citalopram. Pharmacoepidemiol Drug Saf 2005; 14: 367-72.

30.- Martínez JC, Silva $H$ : El año más depresivo de los antidepresivos. Rev GU 2006; 2: 318-28.

31.- Simon G: How can we know whether antidepressants increase suicide risk?. Am J Psychiatry 2006; 163: 1861-3.

32.- Simon G, Savarino J, Operskalski B, Wang P: Suicide risk during antidepressant treatment. Am J Psychiatry 2006; 163: 41-7.

33.- American Academy of Child and Adolescent Psychiatry. Practice parameter for the assessment and treatment of children and adolescents with suicidal behavior. J Am Acad Child Adolesc Psychiatry 2001; 40: 24-51.

34.- Maris R: Suicide. The Lancet 2002; 360: 319-26.

35.- Jick H, Kaye JA, Jick SS: Antidepressants and the risk of suicidal behaviors. JAMA 2004; 292: 338-43.

36.- O'Leary D, Paykel E, Todd C, Vardulaki K: Suicide in primary affective disorders revisited: a systematic review by treatment era. J Clin Psychiatry 2001; 62: 804-11.

37.- Grunebaum MF, Ellis SP, Li S, Oquendo MA, Mann JJ: Antidepressants and suicide risk in the United States, 1985-1999. J Clin Psychiatry 2004; 65: 1456-62.

38.- Olfson M, Shaffer D, Marcus SC, Greenberg T: Relationship between antidepressant medication treatment and suicide in adolescents. Arch Gen Psychiatry 2003; 60: 978-82.

39.- Isacsson G, Bergman U, Rich CL: Epidemiological data suggest antidepressants reduce suicide risk among depressives. J Affect Disord 1996; 41: 1-8.

40.- Isacsson $G$, Boëthius $G$, Bergman $U$ : Low level of antidepressant prescription for people who later commit suicide: 15 years of experience from a population-based drug database in Sweden. Acta Psychiatr Scand 1992; 85: 444-8.

41.- Oquendo MA, Malone KM, Ellis SP, Sackeim HA, Mann JJ: Inadequacy of antidepressant treatment for patients with major depression who are at risk for suicidal behavior. Am J Psychiatry 1999; 156: 190-4.

42.- Luoma JB, Martin CE, Pearson JL: Contact with mental health and primary care providers before suicide: a review of the evidence. Am J Psychiatry 2002; 159: 909-16. 
43.- Gray D, Achilles J, Keller T: Utah youth suicide study, phase I: Government agency contact before death. J Am Acad Child and Adolesc Psychiatry 2002; 41: 427-43

44.- Leon AC, Teres JJ: Paroxetine, other antidepressants, and youth suicide in New York City: 1993 through 1998. J Clin Psychiatry 2004; 65: 915-8.

45.- Isacsson G, Holmgren P, Ahlner J: Selective serotonin reuptake inhibitor antidepressants and the risk of suicide: a controlled forensic database study of 14,857 suicides. Acta Psychiatr Scand 2005; 111: 286-90.

46.- Leon AC, Marzuk PM, Tardiff K, Bucciarelli A, Piper TM, Galea S: Antidepressants and youth suicide in New York City, 1999-2002. J Am Acad Child Adolesc Psychiatry 2006; 45: 1054-8.

47.- Schrader E, Meier B, Brattström A: Hypericum treatment of mild-moderate depression in a placebocontrolled study. Human Psychopharmacol 1998; 3: 163-9.

48.- Tamás Zonda: Depression and Suicidal Behavior. Crisis $2005 ; 26: 34-5$

49.- Gray D, Moskos M, Keller T: Utah Youth Suicide Study new findings, in Proceedings of the 36th Annual Meeting of the American Association of Suicidology. Washington, DC, AAS, 2003.

50.- Isacsson G, Bergman U, Rich CL: Antidepressants, depression and suicide: an analysis of the San Diego study. J Affect Disord 1994; 3: 277-86.

51.- Isacsson $G$, Holmgren P, Druid H, Bergman $U$ : The utilization of antidepressants-a key issue in the prevention of suicide: an analysis of 5281 suicides in Sweden during the period 1992-1994. Acta Psychiatr Scand 1997; 96: 94-100.

52.- Henriksson S, Boethius G, Isacsson G: Suicides are seldom prescribed antidepressants: .ndings from a prospective prescription database in Jamtland county, Sweden, 1985-95. Acta Psychiatr Scand 2001; 103: 301-6.

53.- Marzuk PM, Tardiff K, Leon AC: Use of prescription psychotropic drugs among suicide victims in New York City. Am J Psychiatry 1995; 152: 1520-2.

54.- Ghaemi SN, Ko JY, Goodwin FK: "Cade's Disease" and beyond: Misdiagnosis, antidepressant use, and proposed definition for bipolar spectrum disorder. Can J Psychiatry 2002; 47: 125-34.

55.- Rihmer Z, Szádóczky E, Füredi J, Kiss K, Papp Z: Anxiety disorders comorbidity in bipolar I, bipolar II and unipolar major depression: results from a population-based study in Hungary. J Affect Disord 2001; 67: 175-9.

56.- Wilens TE, Biederman J, Kwon A, et al: A systematic chart review of the nature of psychiatric adverse effects in children and adolescents treated with selective serotonin reuptake inhibitors. J Child Adolesc Psychopharmacol 2003; 13: 143-52.

57.- Faedda GL, Baldessarini RJ, Glovinsky IP, Austin NB: Treatment-emergent mania in pediatric bipolar disorder: a retrospective case review. J Affect Disord 2004; 82: 149-58.

58.- Shia L, Thiebaudb P, McCombsb JS: The impact of unrecognized bipolar disorders for patients treated for depression with antidepressants in the fee-for-services California Medicaid (Medi-Cal) program. J Affect Disord 2004; 82: 373-83.

59.- Hansen L: A critical review of akathisia, and possible association with suicidal behavior. Hum Psychopharmacol Clin Exp 2001; 16: 495-505.

60.- Lane RM: SSRI-induced extrapyramidal side-effects and akathisia: implications for treatment. J Psychopharmacol 1998; 12: 192-214.

61.- Stone TE, Swanson Ch, Feldman MD: Venlafaxine discontinuation syndrome and suicidal ideation A case series. J Clin Psychopharmacol 2007; 27: 94-5.

62.- America Academy of Child and Adolescent Psychiatry. Joint Meeting of the Psychopharmacologic Drugs Advisory Committee, September 28, 2004. http://www. aacap.org/announcements/AACAP_comment.htm. Acceso 20 de marzo 2006. 